\title{
Suppression of tumor-derived Semaphorin 7A and genetic ablation of host-derived Semaphorin 7A impairs tumor progression in a murine model of advanced breast carcinoma
}

\author{
R. GARCIA-AREAS ${ }^{1}$, S. LIBREROS ${ }^{1}$, M. SIMOES ${ }^{1}$, C.CASTRO-SILVA ${ }^{1}$, N. GAZANIGA ${ }^{1}$, \\ S. AMAT ${ }^{1}$, J. JACZEWSKA ${ }^{1}$, P. KEATING ${ }^{1}$, K. SCHILLING ${ }^{2}$, M. BRITO ${ }^{3}$, \\ E.P. WOJCIKIEWICZ ${ }^{1}$ and V. IRAGAVARPU-CHARYULU ${ }^{1}$
}

\author{
${ }^{1}$ Department of Biomedical Sciences, Charles E. Schmidt College of Medicine, Florida Atlantic University; \\ ${ }^{2}$ Lynn Women's Health \& Wellness Institute, ${ }^{3}$ Department of Pathology, \\ Boca Raton Regional Hospital, Boca Raton, FL 33431, USA
}

Received March 27, 2017; Accepted July 31, 2017

DOI: 10.3892/ijo.2017.4144

\begin{abstract}
Solid tumors can generate a plethora of neurogenesisrelated molecules that enhance their growth and metastasis. Among them, we have identified axonal guidance molecule Semaphorin 7A (SEMA7A) in breast cancer. The goal of this study was to determine the therapeutic effect of suppressing SEMA7A levels in the 4T1 murine model of advanced breast carcinoma. We used anti-SEMA7A short hairpin RNA (shRNA) to gene silence SEMA7A in 4T1 mammary tumor cells. When implanted into the mammary fat pads of syngeneic mice, SEMA7A shRNA-expressing 4T1 tumors exhibited decreased growth rates, deferred metastasis and reduced mortality. In vitro, SEMA7A shRNA-expressing 4T1 cells had weakened proliferative, migratory and invasive abilities, and decreased levels of mesenchymal factors. Atomic force microscopy studies showed that SEMA7A shRNA-expressing 4T1 cells had an increase in cell stiffness that corresponded with their decreased malignant potential. Genetic ablation of host-derived SEMA7A further enhanced the antitumor effects of SEMA7A shRNA gene silencing in 4T1 cells. Our preclinical findings demonstrate a critical role for SEMA7A in mediating mammary tumor progression.
\end{abstract}

\section{Introduction}

Semaphorins are a large family of conserved proteins originally characterized as directional cues in axonal guidance and neurite outgrowth in neurogenesis (1-3). Subsequently, it

Correspondence to: Dr Vijaya Iragavarapu-Charyulu, Department of Biomedical Sciences, Charles E. Schmidt College of Medicine, Florida Atlantic University, 777 Glades Road, Boca Raton, FL 33431, USA

E-mail: iragavar@health.fau.edu

Key words: Semaphorin 7A, breast cancer, metastasis, mesenchymal, invasion has been revealed that semaphorins and their receptors carry out roles beyond neurogenesis and serve interesting functions in immune regulation, extracellular matrix remodeling, organogenesis, and angiogenesis (3-7). Studies have identified the expression of Semaphorin 7A (SEMA7A) in various tumor types, however few have described functional roles for SEMA7A in tumor progression (8-11). Hence, its contribution to tumor progression remains relatively unknown in comparison to other vertebrate semaphorins.

SEMA7A, or CD108w, is a 20 -kDa GPI-anchored transmembrane protein expressed by multiple cell types including: neurons, immune cells, melanocytes, fibroblasts, bone cells, and tumor cells (12). This protein can be shed from the cellular membrane by action of ADAM-17(TACE) (13). Both anchored and soluble forms of SEMA7A have been shown to bind to Plexin $C 1$ and $\beta-1$ integrin (CD29) (14-17). The latter activates the MAPK and FAK pathways and causes an increase in proinflammatory cytokines (18). Our group has demonstrated that DA-3 murine mammary tumor cells exhibit high levels of SEMA7A and that suppression of tumor-derived SEMA7A resulted in decreased macrophage-mediated angiogenesis (15).

In this study we further assessed the direct effects of SEMA7A suppression on the highly malignant 4T1 breast carcinoma model. Gene silencing of SEMA7A in 4T1 cells yielded a strong antitumor effect in vivo and SEMA7A shRNA-expressing 4T1 cells showed an impaired ability to proliferate, migrate and invade. These cells also had a decrease in mesenchymal properties, with an increase in cell stiffness. Genetic ablation of host-derived SEMA7A increased the antitumor effects of SEMA7A shRNA. Our study shows a novel functional role for SEMA7A in the progression of mammary tumors.

\section{Materials and methods}

Mice and cell lines. Female BALB/c mice (8-12-week-old) were obtained from Charles River Laboratories, and SEMA7A ${ }^{-1-}$ mice generated by Dr A.L. Kolodkin (Johns Hopkins University, Baltimore, MD, USA), were purchased from 
Jackson Laboratories. Using a speed congenic approach $(19,20)$ SEMA7A $^{-1}$ mice were backcrossed to a BALB/c background, reaching $99.9 \%$ of desired BALB/c background. Mice were housed and used according to the National Institutes of Health guidelines, under protocols approved by Florida Atlantic University Institutional Animal Care and Use Committee. EpH4 mammary cells were provided by Dr Jenifer Prosperi, Indiana University School of Medicine (South Bend, IN, USA). EpH4, 67NR, 4T07, 4T1 and 4T1-LUC (Perkin-Elmer, Waltham, MA, USA) cells were grown in complete DMEM media with $10 \%$ FBS. Female BALB/c or SEMA7A ${ }^{-1}$ $\mathrm{BALB} / \mathrm{c}$ mice were inoculated in the mammary fat pads with $5 \times 10^{5}$ luciferase transfected $4 \mathrm{~T} 1$ or $4 \mathrm{~T} 1-\mathrm{WT}$ mammary tumor cells. Bioluminescent imaging of 4T1-LUC tumor bearers was done up to 3-weeks post-tumor cell implantation. For 4T1 and 4T1-LUC tumor-bearing mice, lungs were collected at 42-days post-tumor cell implantation.

$R N A$ isolation and real-time reverse transcriptase-polymerase chain reaction. Total RNA was extracted from murine or human tumor cells, using the RNeasy Protect Mini kit (Qiagen, Gemantown, MD, USA) according to the manufacturer's instructions. Briefly, cDNA was synthesized using Quantitech Reverse Transcription kit (Qiagen) and gene expression was detected by SYBR Green real-time quantitative polymerase chain reaction (qPCR) analysis using $\mathrm{SYBR}_{\mathrm{RT}} \mathrm{q}^{2} \mathrm{qPCR}$ primers (Qiagen, proprietary primers, sequence not disclosed) from SABioscience (Qiagen). The mRNA levels of gene of interest were normalized to $\beta$-actin, GAPDH or HSP90ab mRNA levels. PCR cycles followed the sequence: $10 \mathrm{~min}$ at $95^{\circ} \mathrm{C}$ of initial denaturation; $15 \mathrm{sec}$ at $95^{\circ} \mathrm{C}$; and 40 cycles of 1 min each at $60^{\circ} \mathrm{C}$ for annealing. The samples were amplified using the Stratagene Mx3005O cycler.

Flow cytometry studies. Ki67 antibodies (Biolegend, San Diego, CA, USA) were used to determine cellular proliferation by flow cytometry according to the manufacturer's protocol. Cells $(50,000)$ were acquired using a FACSCalibur (BD, Franklin Lakes, NJ, USA) flow cytometer, followed by analysis using FloJo software (Tree Star, Inc., Ashland, OR, USA).

Silencing of SEMA7A in $4 T 1$ murine mammary tumor cells. Semaphorin 7A gene silencing in 4T1-LUC mammary tumor cells was achieved using RNA interference via short hairpin RNA. To confirm gene knockdown, qPCR was performed using the SEMA7A specific primers according to the manufacturer's protocol (Qiagen). A SureSilencing shRNA plasmid (Qiagen) with one of two insert sequences was used to target SEMA7A in the 4T1-LUC cells, shRNA1 (ccatagcttt gtcttcaatat) or shRNA2 (cctagctgcatcetgttcatt). Cells were passaged and selected with G418 $(800 \mu \mathrm{g} / \mathrm{ml})$ until at least a 5 -fold decrease in the SEMA7A gene expression was achieved when compared to the scramble shRNA control. For non-luciferase 4T1 cells, an optimized short hairpin RNA algorithm was used to select the top three miRE shRNA sequences targeting SEMA7A (21). The miRE shRNA1 targeted the 5' end of the SEMA7A mRNA (tatgatgataagatctatagtgaagccacagatg tatagatcttatcatcataggcttt) and miRE shRNA2 targeting the 3 ' end of the SEMA7A mRNA (caggagtactagaataatagtgaagc cacagatgtattattctagtactcetgggctat) (Mirimus). As a negative control, we used pooled clones expressing miRE shRNA against Renilla Firefly Luciferase. Cells were transfected with shRNA encoding plasmids using Avalanche transfection reagent (EZ-Biosystems) and selected with puromycin $(3 \mu \mathrm{g} / \mathrm{ml})$. The shRNA vectors also expressed a GFP reporter protein (21). Gene knockdown was confirmed by qPCR using SEMA7A specific primers according to the manufacturer's protocol (Qiagen). The results of gene expression were then confirmed by determination for the SEMA7A protein.

Atomic force microscopy (AFM) cell stiffness measurements. Cell stiffness measurements were acquired on living 4T1 cells. The bare AFM tip was lowered onto the cell surface at $4 \mu \mathrm{m} / \mathrm{s}$ (22). The acquired force-indentation curves of the cells were fit to a model initially proposed by Hertz to estimate the Young's modulus assuming that the cell is an isotropic elastic solid and the AFM tip is a rigid cone (23). The model is as follows:

$$
F=\frac{K}{2\left(1-v^{2}\right)} \frac{4}{\pi \tan \theta} \alpha^{2}
$$

where $F$ is the applied force, $\alpha$ the indentation, $K$ the Young's modulus, $\theta$ the angle formed by the indenter and the plane of the surface $\left(55^{\circ}\right)$ and $v$, Poisson ratio (0.5). Young's modulus was obtained by least square analysis of the force-indentation curves using Igor Pro software.

Migration and invasion assay. 4T1-LUC-Scramble-shRNA cells or 4T1-LUC-SEMA7A-shRNA cells, mammary tumor cells, were cultured under optimal conditions using DMEM culture media with $10 \%$ FBS in 24 wells containing a 500- $\mu \mathrm{M}$ wide culture-insert (Ibidi, Fitchburg, WI, USA) until $\sim 80 \%$ confluency was achieved. Subsequently, the 10\% FBS DMEM was replaced with $0.5 \%$ FBS DMEM and the culture insert was removed to expose cell-free gap. Gap width was assessed at 0,6 and 12-h post-challenge. For the invasion assay, 10,000 serum-starved 4T1-LUC-Scramble-shRNA cells or 4T1-LUC-SEMA7A-shRNA were seeded into a 24-well system of $8-\mu \mathrm{M}$ Transwell inserts coated with Cultrex Basement Membrane Extract (Corning, Tewksbury, MA, USA) and cells were allowed to invade for $18 \mathrm{~h}$. Cells that invaded through insert were dissociated with Tryple Express (Invitrogen, Carlsbad, CA, USA) and stained with Calcein AM. Fluorescence measurements were transformed into cell numbers using a pre-determined cell standard curve and percent invasion was calculated.

Statistical analysis. Results are expressed as means \pm standard deviation. Statistical analyses were performed using GraphPad Prism 6 software (La Jolla, CA, USA). Statistical comparisons were performed using an unpaired 2-tailed Student's t-test, with significance at $\mathrm{P}<0.05$. For multiple comparisons of tumor growth and metastasis, a two-way ANOVA with a Dunn's post hoc test was performed. For analyzing the survival of tumor-bearing mice, the Kaplan-Meier method was used.

\section{Results}

SEMA7A is overexpressed in $4 T 1$ mammary tumor cells and is required for tumor growth in vivo. We evaluated SEMA7A gene 
A

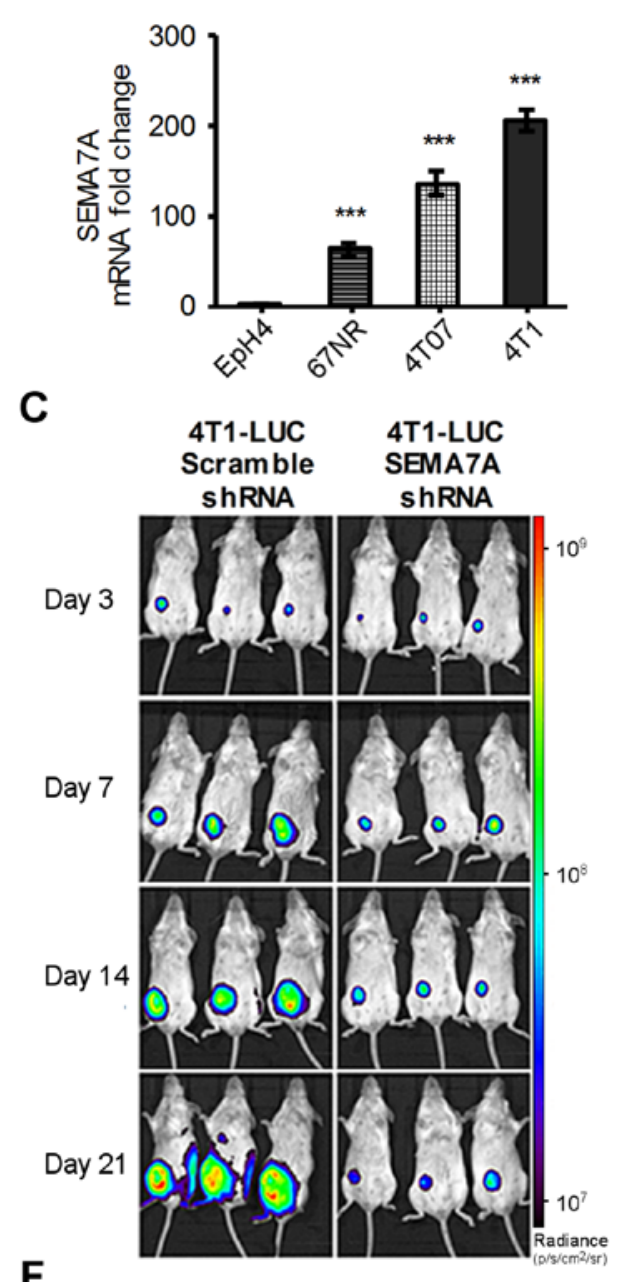

$\mathbf{F}$

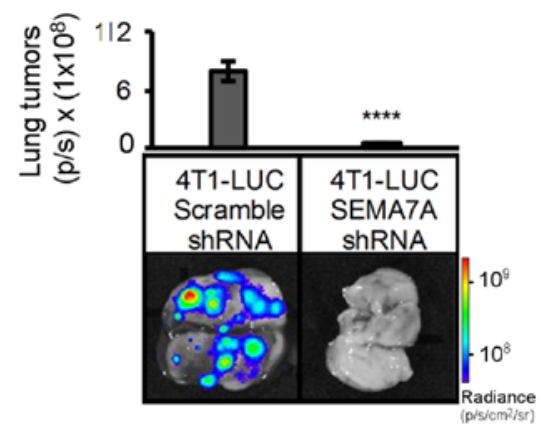

B
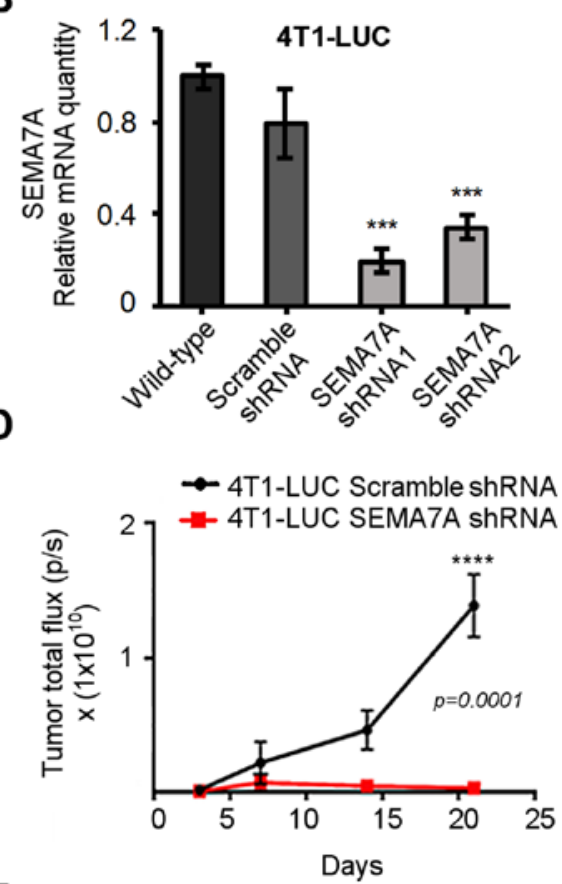

E

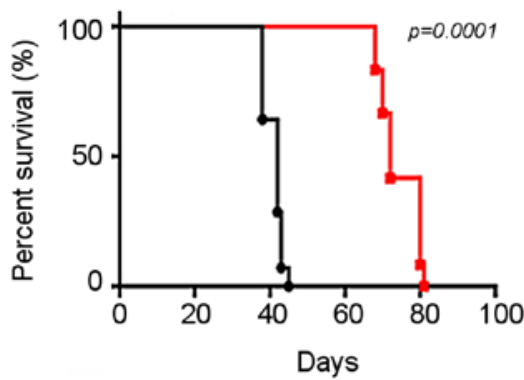

G

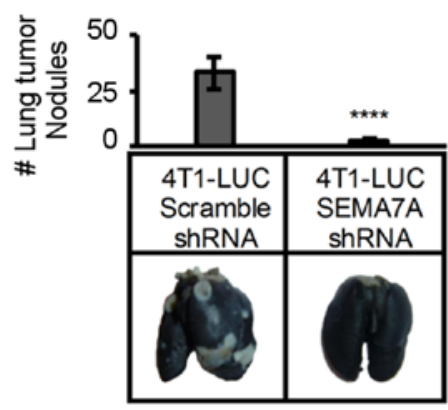

Figure 1. 4T1 cells have high levels of SEMA7A and SEMA7A shRNA gene silencing of 4T1 cells decreases their growth and metastasis in vivo. (A) Transcript analyses of 67NR, 4T07 and 4T1 tumors cells by qPCR, normalized to SEMA7A levels of non-tumorigenic EpH4 mammary epithelia cells ( $\mathrm{n}=3$, unpaired two-tailed Student's t-test). (B) qPCR analyses of SEMA7A mRNA levels in 4T1-LUC-Scramble-shRNA, 4T1-LUC-SEMA7A-shRNA1 and 4T1-LUC-SEMA7A-shRNA2 tumors cells, normalized to SEMA7A levels in 4T1-LUC wild-type (n=3, unpaired two-tailed Student's t-test). (C) 4T1-LUCScramble-shRNA or 4T1-LUC-SEMA7A-shRNA tumor cells were implanted in the mammary fat pads of wild-type female BALB/c, and non-invasive bioluminescent imaging was done at specified time-points and (D) reported as normalized photons $/ \mathrm{sec}$ ( $\mathrm{n}=5$ mice, repeated three times, two-way ANOVA). (E) Kaplan-Meier survival curve of wild-type BALB/c mice bearing 4T1-LUC-Scramble-shRNA or 4T1-LUC-SEMA7A-shRNA tumor cells (n=5 mice, repeated three times, log-rank test). (F) On day 42 post-tumor implantation, lungs were excised from mice bearing 4T1-LUC-Scramble-shRNA or 4T1-LUCSEMA7A-shRNA and imaged for tumor cell-specific bioluminescent signals from lung metastasis nodules $(\mathrm{n}=5$ mice, repeated three times, unpaired two-tailed Student's t-test). (G) India Black ink staining was done to determine number of macro-metastasis lesions, which remained unstained ( $\mathrm{n}=5$ mice, repeated three times, unpaired two-tailed Student's t-test). Data are presented as mean \pm SD. ${ }^{* * *} \mathrm{P} \leq 0.001,{ }^{* * * * *} \mathrm{P} \leq 0.0001$.

expression levels in non-tumorigenic EpH4 murine mammary cells and three sister murine mammary tumor cell lines with varying degrees of malignancy (67NR, 4TO7 and 4T1). 67NR can form slow-growing tumors but fail to disseminate, 4T07 can form tumors that disseminate but cannot metastasize, and 4T1 can form rapid growing tumors that complete all the steps required for metastasis (24). Analyses by qPCR showed that EpH4 cells had nearly undetectable levels of SEMA7A and levels were increased 60-fold in 67NR, 140-fold in 4T07 and 210 -fold in 4T1 tumor cells when compared to that of EpH4 cells (Fig. 1A). These results indicate that as the malignant capabilities of mammary cells increased, so did the SEMA7A 
A

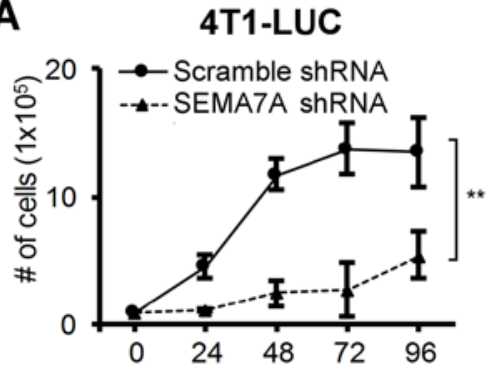

(h)

D

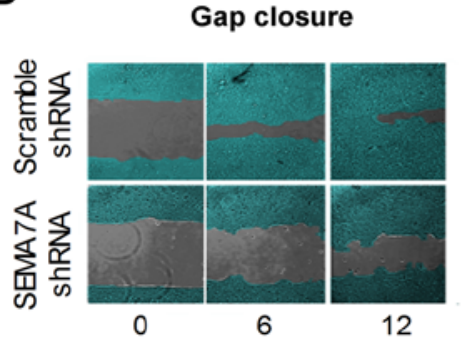

(h)

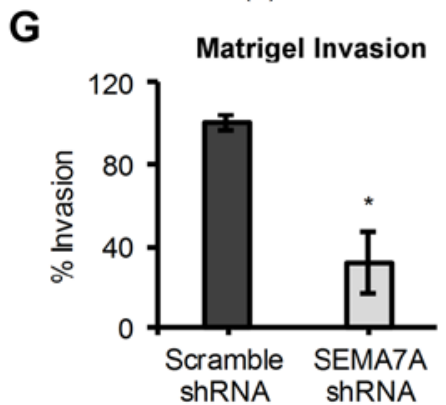

B

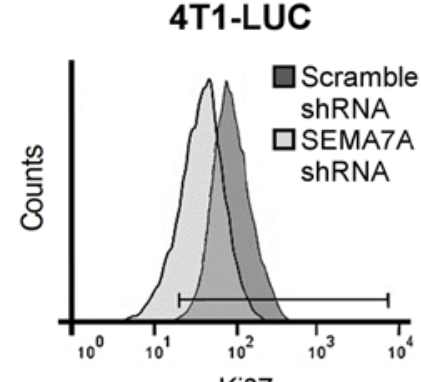

E

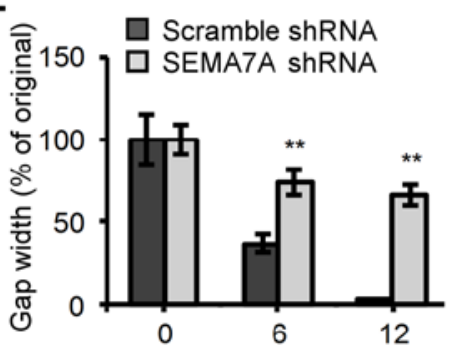

(h)
C 4T1-LUC

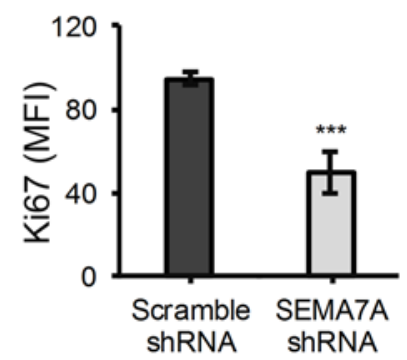

$F$

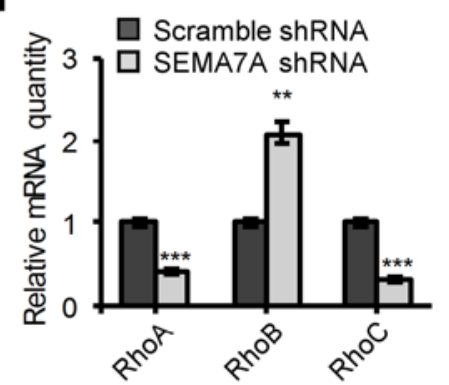

H

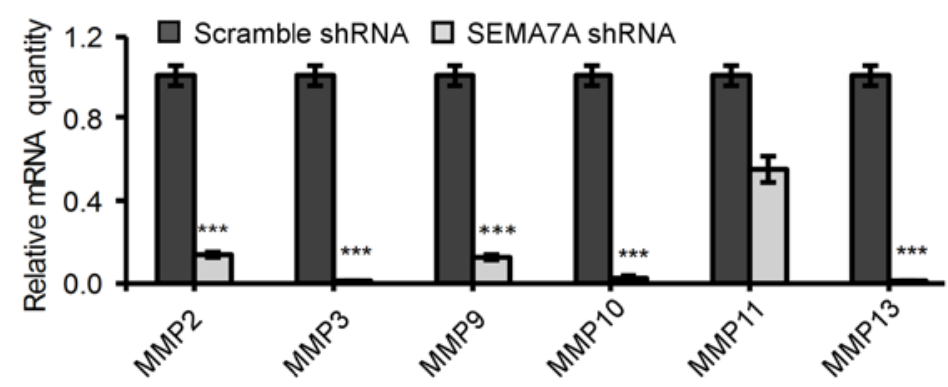

Figure 2. Silencing of SEMA7A gene in 4T1-LUC cells decreases their proliferation, motility and invasion. (A) Growth curves of 4T1-LUC-Scramble-shRNA and 4T1-LUC-SEMA7A-shRNA tumor cells under optimal conditions (n=6, unpaired two-tailed Student's t-test). (B) Flow cytometric analyses of proliferation marker Ki67 in 4T1-LUC-Scramble-shRNA and 4T1-LUC-SEMA7A-shRNA tumor cells. (C) Quantification of Ki67 expression (n=3, unpaired two-tailed Student's t-test). (D) 4T1-LUC-Scramble-shRNA and 4T1-LUC-SEMA7A-shRNA cells were grown to confluency in a 24-well with a culture insert to generate a 500- $\mu \mathrm{M}$ cell-free gap. At time zero, insert was removed and gap-closure was observed at specified time-points and (E) reported as percent width of original gap ( $n=3$, unpaired two-tailed Student's t-test). (F) Gene expression of RhoA, RhoB and RhoC was assayed by qPCR in 4T1-LUC-SEMA7A-shRNA tumor cells, normalized to 4T1-LUC-Scramble-shRNA ( $\mathrm{n}=3$, unpaired two-tailed Student's t-test). (G) 4T1-LUC-Scramble-shRNA and 4T1-LUC-SEMA7A-shRNA cells' in vitro invasion through a Matrigel coated $8 \mu \mathrm{M}$ Transwell, reported as percent invasion using 4T1-LUC-Scramble-shRNA cells as 100\% (n=3, unpaired two-tailed Student's t-test). (H) Gene expression of specified MMPs was assayed by qPCR in 4T1-LUC-SEMA7A-shRNA tumor cells, normalized to levels of 4T1-LUC-Scramble-shRNA cells ( $\mathrm{n}=3$, unpaired two-tailed Student's t-test). Data are presented as mean $\pm \mathrm{SD}$. ${ }^{*} \mathrm{P} \leq 0.05$, ${ }^{* *} \mathrm{P} \leq 0.01,{ }^{* * * *} \mathrm{P} \leq 0.001$.

levels. We therefore posed the question: does SEMA7A have a functional role in mammary tumor cell malignancy? We strategized to use shRNA to suppress the levels of SEMA7A in the highly aggressive 4T1 cells and assess its effects in vivo. We chose to use 4T1 cells expressing luciferase (4T1-LUC) as they enabled us to perform non-invasive bioluminescent imaging to monitor tumor progression. SEMA7A levels were equivalent in 4T1-LUC cells and wild-type 4T1 cells (data not shown). Experimental control cells were generated by transfecting 4T1-LUC cells with a plasmid encoding for a non-targeting scramble shRNA (4T1-LUC-Scramble-shRNA). 4T1-LUC wild-type and 4T1-LUC-Scramble-shRNA cells had comparable SEMA7A levels (Fig. 1B). 4TI-LUC cells were also transfected with plasmids encoding for one of four SEMA7A shRNA sequences. Two of the shRNA strands targeting SEMA7A showed consistent silencing efficiency, 4T1-LUC-SEMA7A-shRNA1 and 4T1-LUCSEMA7A-shRNA2 (Fig. 1B). 4T1-LUC-SEMA7A-shRNA1 had an $80 \%$ reduction in SEMA7A levels compared to both 4T1-LUC wild-type cells and 4T1-LUC-Scramble-shRNA cells, while 4T1-LUC-SEMA7A-shRNA2 had a 55\% reduction (Fig. 1B). Given its enhanced silencing efficiency, we continued our studies using the 4T1-LUC-SEMA7A-shRNA1 clone, which will be referred from here on as 4T1-LUCSEMA7A-shRNA. The commercially available anti-SEMA7A murine antibodies at the time of this study were determined to be unspecific in our hands. The antibodies tested generated false positives when used for western blotting and immunofluorescence analysis of SEMA7A-shRNA silenced 4T1 cells and tissue samples from SEMA7A-/- mice. Hence, we utilized SEMA7A mRNA as an evaluative readout in this study in order to effectively differentiate its levels from that of other Semaphorins.

We implanted 4T1-LUC-Scramble-shRNA or 4T1-LUCSEMA7A-shRNA tumor cells into the mammary fat pads of syngeneic wild-type BALB/c mice and bioluminescent 
imaging was performed to monitor tumor growth (Fig. 1C). 4T1-LUC-SEMA7A-shRNA tumor bearers displayed a significant reduction $(\mathrm{P}=0.00001)$ of tumor burden (Fig. 1D) and a significant increase $(P=0.00001)$ in survival (Fig. 1E). We questioned whether the reduced tumor burden in 4T1-LUCSEMA7A-shRNA tumor-bearing mice could have changed the kinetics of metastatic dissemination. We surveyed the lungs of tumor bearers at day 42 post-tumor implantation given that $4 \mathrm{~T} 1$ tumor cells are known to metastasis primarily to the lung (25). Detection of the luciferase signals revealed a significant $\sim 80 \%$ decrease $(\mathrm{P}=0.00001)$ of tumor cell-specific bioluminescence in the lungs of 4T1-LUCSEMA7A-shRNA tumor bearers (Fig. 1F). Enumeration of the metastatic nodules, which remain unstained after India Black staining, revealed $>30$ metastatic foci in mice bearing 4T1-LUC-Scramble-shRNA cells, compared to $<5$ metastatic foci in lungs of 4T1-LUC-SEMA7A-shRNA mammary tumor bearers (Fig. 1G). The lung metastasis nodules that eventually developed in 4T1-LUC-SEMA7A-shRNA tumor-bearing mice retained SEMA7A expression (data not shown), indicating they arose from tumor cells in which SEMA7A gene expression had either never been suppressed or that had lost the suppression. Taken together, our results indicate that SEMA7A produced by tumor cells is actively involved in mammary tumor progression.

SEMA7A shRNA gene silencing decreases the proliferative, migratory and invasive potential of $4 T 1$ cells. Given that tumor growth was impaired in 4T1-LUC-SEMA7A-shRNA mammary tumor-bearing mice (Fig. 1), we sought to examine the effects of shRNA-mediated suppression of SEMA7A on 4T1 cells in vitro. We first observed a reduced growth rate in 4T1-LUC-SEMA7A-shRNA tumor cells (Fig. 2A) that was confirmed by assaying for the expression of the proliferation marker Ki67 by flow cytometry (Fig. 2B). We found a decrease of nearly half the expression of Ki67 in 4T1-LUC-SEMA7A-shRNA tumor cells compared to control (Fig. 2C). Next, we challenged 4T1-LUC-Scramble-shRNA and 4T1-LUC-SEMA7A-shRNA cells to populate a $500-\mu \mathrm{M}$ cell-free gap under serum-low conditions in order to assess their migratory potential in vitro (Fig. 2D). At 12-hour postchallenge, 4T1-LUC-Scramble-shRNA cells succeeded in closing the gap but 4T1-LUC-SEMA7A-shRNA only populated $\sim 30 \%$ of the original cell-free gap (Fig. 2E). Given their reduced migratory ability, we surveyed if SEMA7A gene silencing had also affected the levels of important regulators of cell migration: RhoA/B/C (26). When compared to 4T1-LUCScramble-shRNA cells, 4T1-LUC-SEMA7A-shRNA cells exhibited decreased levels of migration/motility promoting RhoA and RhoC but increased levels of tumor-limiting RhoB (Fig. 2F). We next questioned whether decreased SEMA7A levels could also affect the invasiveness of 4T1 cells. 4T1-LUC-Scramble-shRNA and 4T1-LUC-SEMA7A-shRNA tumor cells were labeled with Calcein AM and seeded into the upper chamber of a Matrigel coated, $8-\mu \mathrm{M}$ pore Transwell insert. After $12 \mathrm{~h}$, we quantified the percentage of Calcein AM-positive cells that were able to invade through the Matrigel-coated insert and into the lower chamber of the well. We found that 4T1-LUC-SEMA7A-shRNA tumor cells had a $\sim 60 \%$ significant reduction in invasion $(\mathrm{P} \leq 0.05)$ when compared to 4T1-LUC-Scramble-shRNA tumor cells (Fig. 2G). To further determine the effect of SEMA7A on invasive abilities, we surveyed for the levels of various matrix metalloproteinases (MMPs) that have been determined critical in mediating tumor cell invasion (27). Matrix metalloproteinases MMP-2, -3, -9, -10 and -13 were significantly $(\mathrm{P} \leq 0.001)$ decreased in 4T1-LUC-SEMA7A-shRNA tumor cells, suggesting a strong linkage between the gene expression of SEMA7A and levels of MMPs (Fig. 2H). Our results indicate that suppression of tumor-derived SEMA7A weakens the ability of 4T1 cells not only to proliferate, but also to migrate and invade. These changes in proliferative, migratory and invasive potentials may partially account for the decreased tumor progression exhibited by 4T1-LUC-SEMA7A-shRNA tumor cells in vivo (Fig. 1).

Decreased SEMA7A levels in $4 T 1$ mammary tumor cells promotes an epithelial-like morphology and decreased levels of mesenchymal-promoting factors. It has been shown that gain of mesenchymal properties in 4T1 cells may lead to increased invasive behavior in vivo (28) and that expression of SEMA7A has been shown to promote a mesenchymal phenotype in cells (9). We therefore questioned if SEMA7A gene silencing had altered the epithelial/mesenchymal properties of 4TI-LUC cells. 4T1-LUC-SEMA7A-shRNA cells displayed an enhanced epithelial-like morphology with cells growing in tight, rounded colonies (Fig. 3A). This change towards an epithelial-like morphology led us to probe whether silencing of SEMA7A in 4T1-LUC-SEMA7A-shRNA cells may have tilted the levels of mesenchymal-promoting factors. We first assessed TGF- $\beta 1$ levels as it has been shown to be a key mediator of mesenchymal programs (29) and has been tightly linked to SEMA7A (17). We found that TGF- $\beta 1$ levels were significantly decreased $(\mathrm{P} \leq 0.001)$ in 4T1-LUC-SEMA7A-shRNA cells when compared to 4T1-LUC-Scramble-shRNA cells, but no significant changes were detected in the levels of TGF- $\beta 2$ and TGF- $\beta 3$ (Fig. 3B). 4T1-LUC-SEMA7A-shRNA cells also had a marked decrease in the expression of known TGF- $\beta 1$ induced mesenchymal promoting factors: Snail1, Snail2 and Twist (30) (Fig. 3C). In addition, 4T1-LUC-SEMA7A-shRNA cells had decreased levels of ZEB1 and ZEB2 compared to 4T1-LUCScramble-shRNA (Fig. 3D), both of which are strong inducers of a mesenchymal phenotype and correlated with enhanced aggressive behavior in tumor cells (31). Given that Snail1 has been shown to induce the expression of mesenchymal markers Vimentin (VIM) and N-cadherin (CDH2) (32), we assayed for the expression of these markers in 4T1 cells upon SEMA7A gene silencing. 4T1-LUC-SEMA7A-shRNA cells showed an $\sim 90 \%$ decrease in Vimentin levels and an $\sim 80 \%$ decrease in N-cadherin levels when compared to 4T1-LUCScramble-shRNA cells (Fig. 3E). Vimentin and N-cadherin levels have been shown to be inversely correlated with E-cadherin (CDH1) (33). We sought to determine if SEMA7A gene silencing could have affected the E-cadherin (CDH1) levels, but we found they remained unchanged between 4T1-LUC-Scramble-shRNA and 4T1-LUC-SEMA7A-shRNA cells (Fig. 3F). Like E-cadherin, tight-junction marker Desmoplakin (DSP) has also been shown to be lost during the epithelial to mesenchymal transition (32). We found a 
A

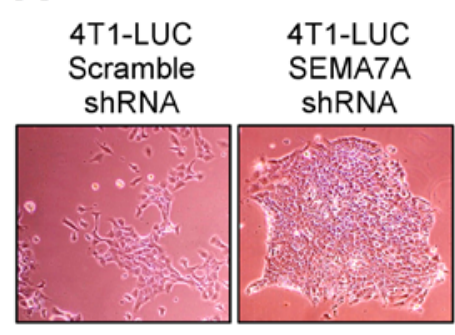

B

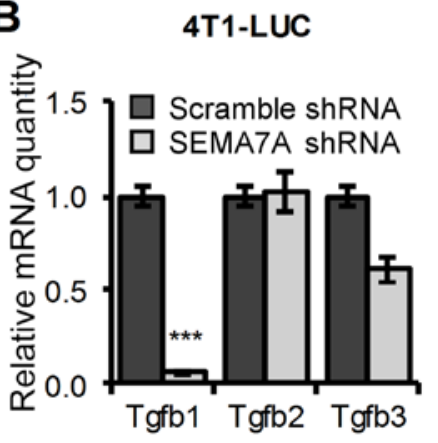

C

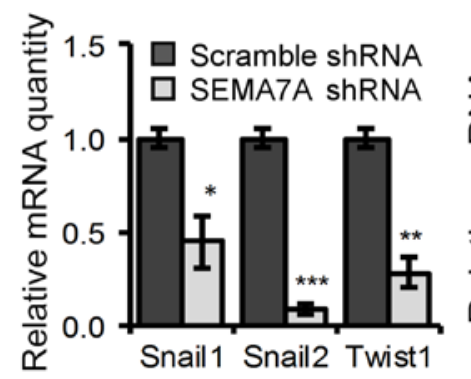

D

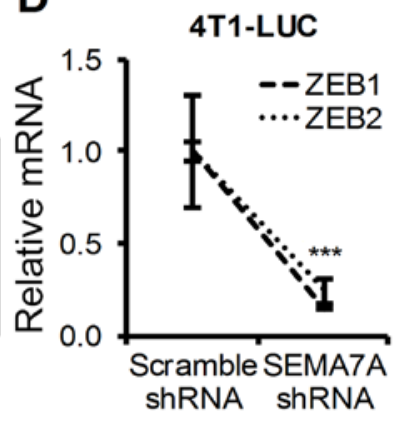

E

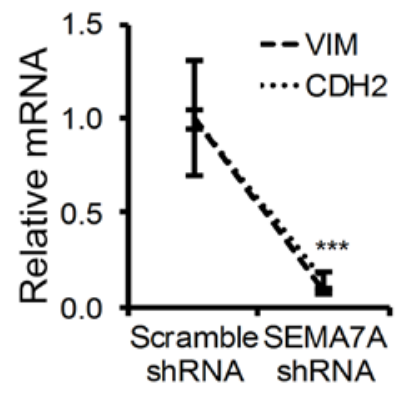

$\mathbf{F}$

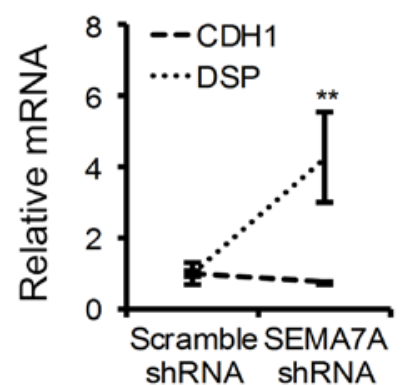

Figure 3. SEMA7A shRNA gene silencing in 4T1-LUC cells changes cell morphology, decreases gene expression of EMT-inducing genes and increases Desmoplakin gene expression. (A) SEMA7A gene-silenced 4T1-LUCSEMA7A-shRNA cells exhibited compact round colonies when compared to 4T1-LUC-Scramble-shRNA cells as observed by phase contrast imaging (images representative of 15 fields of view, 20x magnification). (B-F) Gene expression levels of specified genes (TGF $\beta$; Snail and Twist; ZEB1 and ZEB2; Vimentin (VIM) and N-cadherin (CDH2); E-cadherin (CDH1) and Desmoplakin (DSP) of 4T1-LUC-SEMA7A-shRNA tumor cells, normalized to levels in 4T1-LUC-Scramble-shRNA ( $\mathrm{n}=3$, unpaired two-tailed Student's $\mathrm{t}$-test). Data are presented as mean $\pm \mathrm{SD} .{ }^{*} \mathrm{P} \leq 0.05,{ }^{* *} \mathrm{P} \leq 0.01,{ }^{* * *} \mathrm{P} \leq 0.001$.

significant 5-fold increase $(\mathrm{P} \leq 0.01)$ of Desmoplakin levels in 4T1-LUC-SEMA7A-shRNA cells compared to 4T1-LUCScramble-shRNA cells (Fig. 3F). Our results indicate that shRNA silencing of the SEMA7A gene in 4T1 cells led to a shift towards a more epithelial morphology, with decreased levels of mesenchymal markers that have been linked to enhanced tumor growth and metastasis.

SEMA7A gene silencing increases stiffness of $4 T 1$ cells. Atomic force microscopy (AFM) has recently been shown to be useful in distinguishing malignant cells from normal cells (34). Analyses of biomechanical properties with AFM has shown that aggressive cancerous cells are less stiff compared to normal cells in $2 \mathrm{D}$ cultures $(35,36)$. AFM measurements were acquired to determine the relative stiffness of

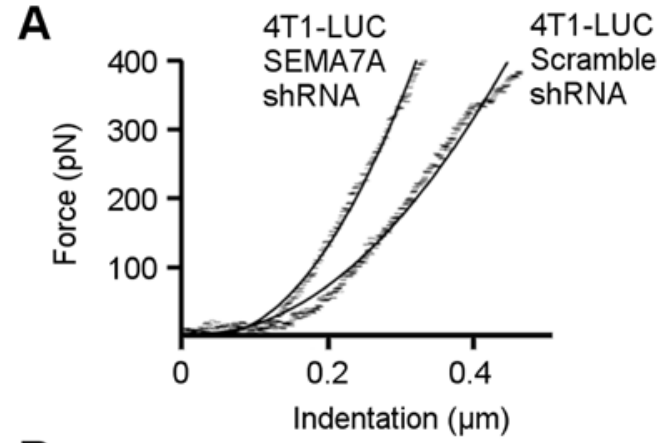

B

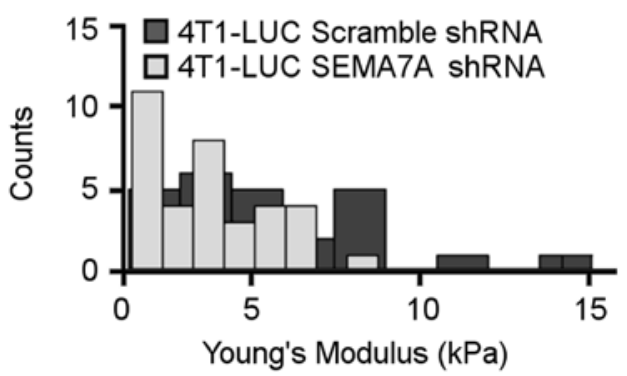

C

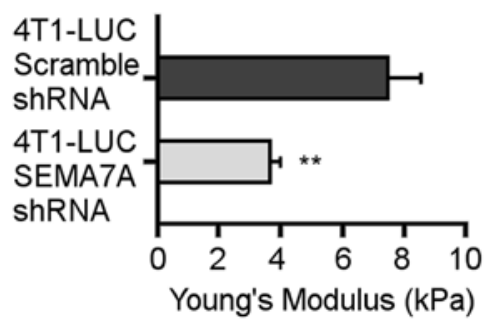

Figure 4. SEMA7A alters tumor cell stiffness. (A) Representative forceindentation curves from AFM cell stiffness measurements acquired for 4T1-LUC-Scramble-shRNA and 4T1-LUC-SEMA7A-shRNA cells. Fitted curves derived from the Hertz model are overlaid on the raw data. AFM measurements were acquired at $37^{\circ} \mathrm{C}$ at a constant cantilever retraction rate, applied force and contact time. (B) Data distribution of Young's modulus values for 4T1-LUC-Scramble-shRNA cells $(n=35)$ and 4T1-LUCSEMA7A-shRNA cells $(n=29)$. (C) Average of Young's modulus values for stiffness measurements from (B). Data are presented as mean \pm SEM. ${ }^{* * *} \mathrm{P} \leq 0.01$.

4T1-LUC-Scramble-shRNA and 4T1-LUC-shRNA-SEMA7A cells. The AFM cantilever was used as a microindenter, probing the cell $<1 \mu \mathrm{m}$ using applied forces of $<1 \mathrm{nN}$ so as to not damage the cell. Fig. 4A shows representative forceindentation curves acquired for 4T1-LUC-Scramble-shRNA and 4T1-LUC-shRNA-SEMA7A cells. 4T1-LUC-shRNASEMA7A cells indented less than 4T1-LUC-Scramble-shRNA cells for equivalent applied forces. One hundred forceindentation curves were acquired for each cell measured and fitted to the Hertz's model. Histograms in Fig. 4B reveal the data distribution of the Young's modulus values for both cell types. The average Young's modulus value calculated for the 4T1 mammary tumor cells was $3.7 \pm 0.3 \mathrm{kPa}(\mathrm{n}=35)$ (Fig. 4C). Following SEMA7A gene knockdown, cell stiffness increased to $7.5 \pm 1 \mathrm{kPa}(\mathrm{n}=29)$ in $4 \mathrm{~T} 1-\mathrm{LUC}-\mathrm{Scramble}-\mathrm{shRNA}$ cells. Our AFM data supports the notion that an increase in cell stiffness in vitro is inversely related to the malignant behavior of tumor cells, as we observed that the stiffer 4T1-LUC-shRNA-SEMA7A cells had reduced malignant potential in vivo (Fig. 1). 
A

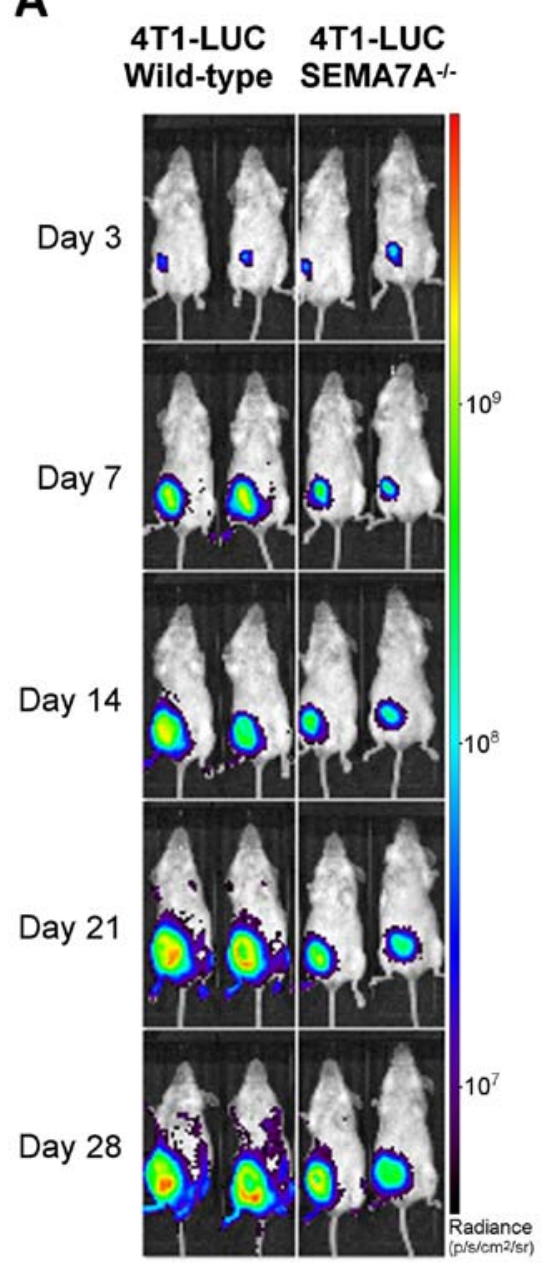

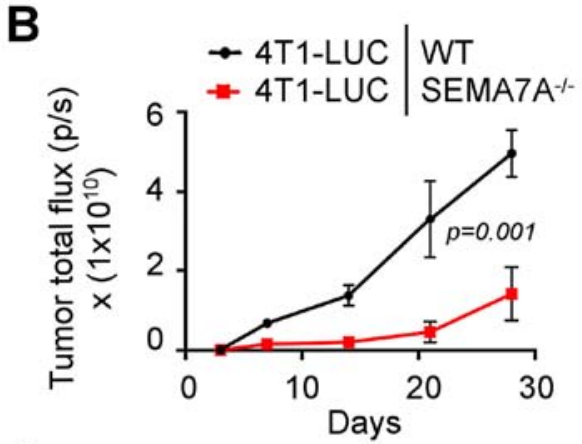

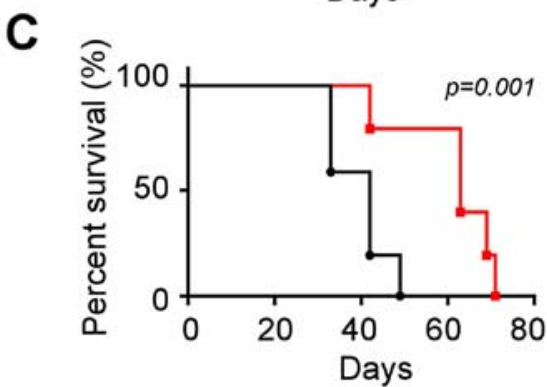

D

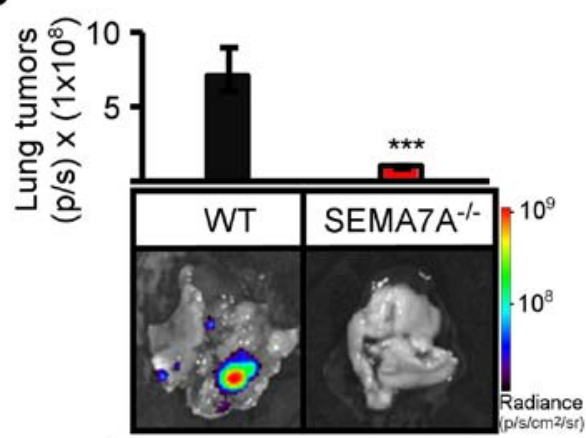

Figure 5. Genetic ablation of host-derived SEMA7A decreases tumor growth rate and metastasis in 4T1-LUC tumor-bearing mice. (A) 4T1-LUC wild-type tumor cells were implanted in the mammary fat pads of wild-type female BALB/c or SEMA7A ${ }^{-/}$BALB/c mice, non-invasive bioluminescent was done at specified time-points and (B) reported as normalized photons/sec ( $\mathrm{n}=5$ mice, repeated three times, two-way ANOVA). (C) Kaplan-Meier survival curve of wild-type BALB/c and SEMA7A ${ }^{-1-}$ BALB/c bearing 4T1-LUC cells ( $n=5$ mice, repeated three times, log-rank test). (D) On day 42 post-tumor implantation, lungs were excised from wild-type BALB/c and SEMA7A ${ }^{-/}$BALB/c bearing 4T1-LUC cells for tumor cell-specific bioluminescent signals from metastasis nodules ( $\mathrm{n}=5$ mice, repeated three times, unpaired two-tailed Student's t-test). Data are presented as mean \pm SD. ${ }^{* * *} \mathrm{P} \leq 0.001$.

Ablation of host-derived SEMA7A impairs tumor growth and enhances the antitumor effects of shRNA suppression of tumor-derived SEMA7A. Given that host cells also express SEMA7A $(14,17,18,37)$, we questioned if genetic ablation of host-derived SEMA7A could further augment the antitumor effects of SEMA7A gene silencing in 4T1 cells. We first determined the effects of solely ablating host-derived SEMA7A on mammary tumor growth. Mammary pads of wild-type or SEMA7A ${ }^{-/}$female BALB/c mice were inoculated with wild-type 4T1-LUC cells. Bioluminescent imaging was performed for 28 days. SEMA7A ${ }^{-/-}$tumor-bearing mice displayed a significant decrease $(\mathrm{P}=0.001)$ in tumor growth rate (Fig. 5A and $\mathrm{B}$ ), increase in survival $(\mathrm{P}=0.001)$ (Fig. 5C), and a decrease in metastasis to the lungs ( $\mathrm{P} \leq 0.001)$ (Fig. 5D).

We next tested the effects of ablating host-derived SEMA7A in addition to suppressing tumor-derived SEMA7A. Towards this, we used an optimized miR-E shRNA system that provided enhanced stable gene knockdown (21). Wild-type 4T1 were transfected with a vector encoding for an shRNAmir targeting the 5' end of the SEMA7A mRNA (4T1-SEMA7A-shRNA1) or one targeting the $3^{\prime}$ end of the
SEMA7A mRNA (4T1-SEMA7A-shRNA2). 4T1-LUC cells could not be used with the miR-E system, as puromycin had been used to select for luciferase expression. To generate an experimental control, 4T1 cells were transfected with a vector encoding for an shRNAmir targeting Renilla luciferase (4T1-Renilla-shRNA). 4T1-Renilla-shRNA cells had SEMA7A levels equivalent to that of wild-type 4T1 cells (data not shown). Both SEMA7A shRNAmirs achieved a $>80 \%$ decrease in SEMA7A levels when compared to 4T1-Renilla-shRNA cells (Fig. 6A). 4T1-Renilla-shRNA cells, 4T1-SEMA7A-shRNA1 or 4T1-SEMA7A-shRNA2 cells were implanted into the mammary fat pads of either wild-type or SEMA7A ${ }^{-/-}$female BALB/c mice. Gene silencing of SEMA7A in 4T1 cells resulted in a significant reduction $(\mathrm{P} \leq 0.0001)$ in tumor burden, but ablation of host-derived SEMA7A further decreased tumor growth by an additional $\sim 15 \%$ (Fig. 6B). At day 42, lungs were excised from tumorbearing mice and assessed for metastatic lesions (Fig. 6C). Ablating host-derived SEMA7A yielded an additional $30 \%$ significant reduction $(\mathrm{P} \leq 0.0001)$ in the number of metastatic lung lesions compared to suppressing tumor-derived SEMA7A 
A

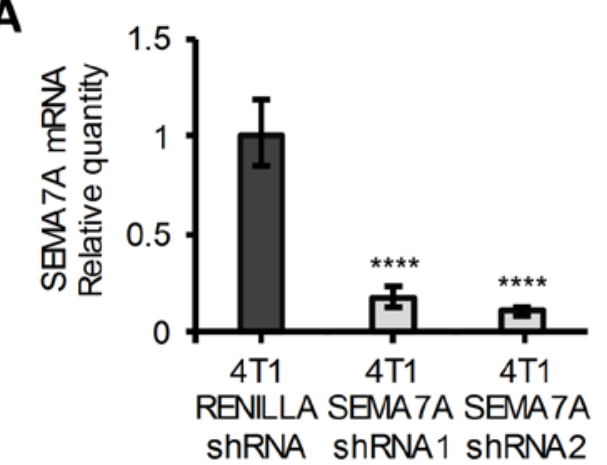

C

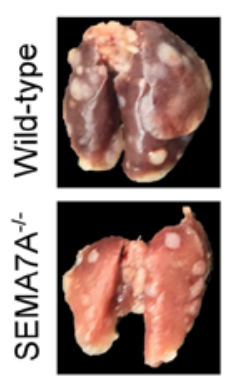

4T1

RENILLA

shRNA

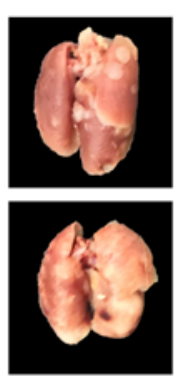

4T1

SEMA7A

shRNA1
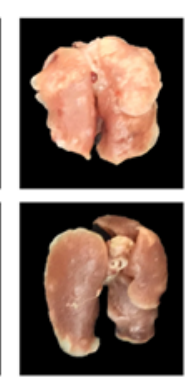

4T1

SEMA7A

shRNA2
B

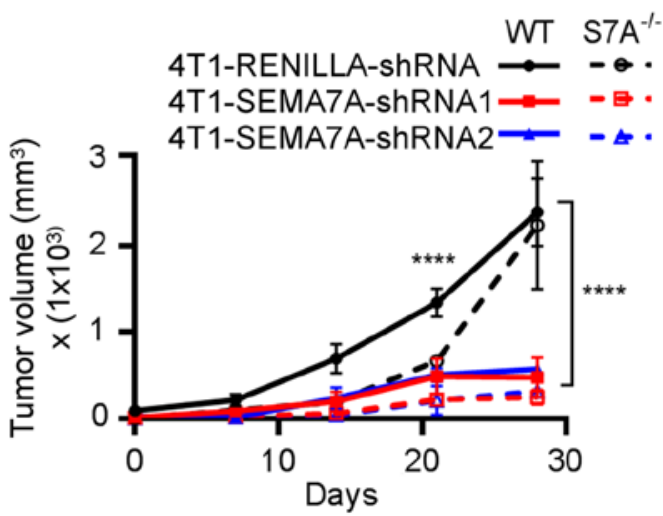

D

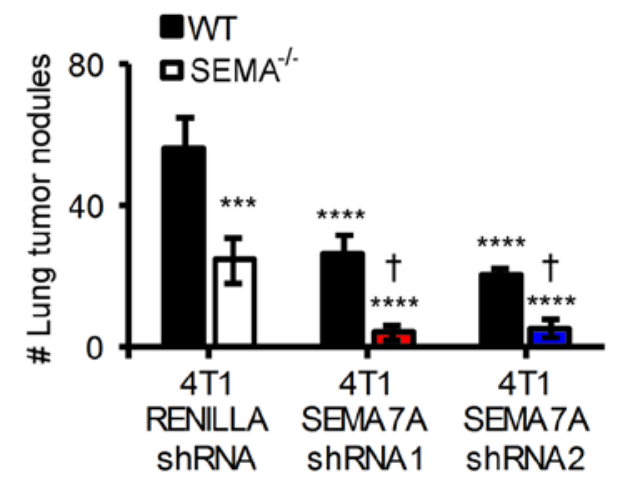

Figure 6. Inhibition of host-derived and tumor-derived SEMA7A decreases rate of tumor growth and metastasis in 4T1 tumor-bearing mice. (A) qPCR analyses of SEMA7A mRNA levels in 4T1-SEMA7A-shRNA1 (targeting 5' end of SEMA7A mRNA) and 4T1-SEMA7A-shRNA2 (targeting 3' end of SEMA7A mRNA) tumors cells, normalized to SEMA7A levels of 4T1-Renilla-shRNA (non-target control) (n=3, unpaired two-tailed Student's t-test). (B) 4T1-LUCScramble-shRNA, 4T1-LUC-SEMA7A-shRNA1 or shRNA2 tumor cells were implanted in the mammary fat pads of wild-type BALB/c or SEMA7A ${ }^{-/-}$BALB/c mice and tumor volume was measured at specified time-points ( $\mathrm{n}=5$ mice, repeated three times, two-way ANOVA. (C) Photographic images of excised and $4 \%$ PFA fixed lungs at day 42 post-tumor implantation, and (D) quantification of macro-metastatic lesions in the lungs at day 42 post-tumor implantation (n=5 mice, repeated three times, two-way ANOVA). Data are presented as mean \pm SD. To represent statistical significance, asterisks denote differential significance of values compared to that of 4T1-Renilla-shRNA/WT-BALB/c and a dagger denotes differential significance compared to $4 \mathrm{~T} 1-$ Renilla-shRNA/SEMA7A ${ }^{-1}$. ${ }^{*} * * * 0.001,{ }^{* * * * *} \mathrm{P} \leq 0.0001,{ }^{\dagger} \mathrm{P} \leq 0.0001$.

alone (Fig. 6D). Our results show that ablation of host-derived SEMA7A and tumor-derived SEMA7A can significantly improve outcomes in our breast carcinoma model.

\section{Discussion}

The objective of this study was to elucidate the role of SEMA7A in breast cancer. To do so, we utilized the 4T1 murine model of advanced breast carcinoma. We found that shRNA suppression of SEMA7A in 4T1 mammary tumor cells significantly inhibited tumor growth, which in turn deferred metastasis and increased survival. When we implanted wild-type 4T1 cells in SEMA7A-deficient mice, we found that lack of host-derived SEMA7A further decreased tumor growth. However, shRNA inhibition of tumor-derived SEMA7A resulted in a greater decrease of tumor burden than genetic ablation of host-derived SEMA7A. When we combined both approaches, the antitumor effects of SEMA7A shRNA were augmented. We will further delineate the contributions of tumor-derived SEMA7A versus that of host-derived SEMA7A. In addition, determining if there are any inherent variations between tumor-specific and host-derived SEMA7A could be useful when designing inhibitory strategies. To date, no tumor-enhancing mutations or variations of SEMA7A have been described.
In addition to breast cancer, SEMA7A has also been shown to be expressed in melanoma, glioblastoma and oral squamous cell carcinoma $(8,11,15,38)$. Multiple studies $(9,10,15,38)$ suggest a strong linkage between SEMA7A and the potential of tumor cells to proliferate, migrate and invade. In terms of invasive potential, 4T1-LUC-shRNA-SEMA7A cells had decreased levels of MMP-2,-3,-9,-10 and -13. Encouragingly, oral squamous cell carcinoma cell lines shRNA gene silenced for SEMA7A displayed decreased MMP-2, -9 and MT1-MMP (38). Hence, these overlapping findings suggest a conserved role for SEMA7A in mediating pro-migration and pro-metastatic MMPs among different cancers.

Further characterization of 4T1-LUC-shRNA-SEMA7A cells in our study revealed decreased levels of mesenchymal promoting factors: Snail, Twist, ZEB1 and ZEB2. Additional studies will determine the specific pathway(s) by which SEMA7A affects the levels of these factors. We speculate that SEMA7A induction of TGF- $\beta$ may play a critical role in promoting a mesenchymal phenotype, as TGF- $\beta$ has been shown to directly induce EMT-promoting transcription factors (32). Supporting the role of SEMA7A in promoting mesenchymal phenotypes, it has been shown that SEMA7A can serve as a differentiation marker for mesenchymal stem cells (39).

It is proposed that gain of mesenchymal properties causes a decrease in cell stiffness (40). A decrease in stiffness allows 
cells to spread more easily on a substrate and thus in turn could facilitate migration and invasion. Transformation of non-metastatic human breast cancer cells into metastatic also caused a decrease in cell stiffness in 2D cultures (41). Our results support the notion that cell stiffness and malignant behavior can be inversely related. Microindentation with an AFM probe showed that 4T1-LUC-shRNA-SEMA7A cells have increased cell stiffness compared to SEMA7A-expressing 4T1 cells. Both in vitro and in vivo, these stiffer cells showed lessened invasion potential. A recent study used AFM to show that SEMA7A decreases the adhesion strength of dendritic cells to the extracellular matrix (42). It would be interesting to know if inhibition of SEMA7A in tumor cells affects their ability to adhere to the extracellular matrix. A potential increase in adhesion to the ECM, coupled with the decreased levels of MMPs we observed, could potentially hinder the ability of tumor cells to migrate and disseminate.

We and others now show that shRNA inhibition of SEMA7A in tumor cells lessens their malignant potential $(10,15,38)$. Although useful in delineating the function of SEMA7A in tumor progression, shRNA has limited therapeutic potential. In order to translate these preclinical findings into therapies, the development of SEMA7A inhibitors will be critical. To date, there are no known agents that specifically target SEMA7A. Black et al corroborated that inhibiting SEMA7A in breast cancer would be beneficial as SEMA7A levels correlated with poor prognosis in breast cancer patients (10).

Overall, our collective results support our hypothesis that SEMA7A promotes breast cancer progression. Our findings postulate a novel role for SEMA7A in breast cancer that may lead to further findings of therapeutic value.

\section{Acknowledgements}

This study was supported by the National Institutes of Health grants NIH R15 CA135513-01 and R15 CA135513-01-OS1, and by the Boca Raton Regional Hospital Foundation. We remember and thank the late Kathy Tabor-McEwan, M.D., for her instrumental efforts in establishing the FAU-BRRH alliance to support this study and her commitment to further breast cancer research.

\section{References}

1. Pasterkamp RJ and Kolodkin AL: Semaphorin junction: Making tracks toward neural connectivity. Curr Opin Neurobiol 13 . 79-89, 2003.

2. Zhou Y, Gunput RA and Pasterkamp RJ: Semaphorin signaling: Progress made and promises ahead. Trends Biochem Sci 33: 161-170, 2008

3. Múzes G and Sipos F: Relation of immune semaphorin/plexin signaling to carcinogenesis. Eur J Cancer Prev 23: 469-476, 2014.

4. Epstein JA, Aghajanian H and Singh MK: Semaphorin signaling in cardiovascular development. Cell Metab 21: 163-173, 2015.

5. Garcia-Areas R, Libreros S and Iragavarapu-Charyulu V: Semaphorin7A: Branching beyond axonal guidance and into immunity. Immunol Res 57: 81-85, 2013.

6. Ito D, Nojima S and Kumanogoh A: The role of Semaphorin family in immune systems. Nihon Rinsho Meneki Gakkai Kaishi 37 1-10, 2014 (In Japanese).

7. Morihana $T$ and Kumanogoh A: Immune semaphorins and allergic diseases. Arerugi 62: 155-162, 2013 (In Japanese).
8. Ma B, Herzog EL, Lee CG, Peng X, Lee CM, Chen X, Rockwell S, Koo JS, Kluger H, Herbst RS, et al: Role of chitinase 3-like-1 and Semaphorin 7a in pulmonary melanoma metastasis. Cancer Res 75: 487-496, 2015

9. Allegra M, Zaragkoulias A, Vorgia E, Ioannou M, Litos G, Beug $\mathrm{H}$ and Mavrothalassitis G: Semaphorin-7a reverses the ERF-induced inhibition of EMT in Ras-dependent mouse mammary epithelial cells. Mol Biol Cell 23: 3873-3881, 2012.

10. Black SA, Nelson AC, Gurule NJ, Futscher BW and Lyons TR: Semaphorin 7 a exerts pleiotropic effects to promote breast tumor progression. Oncogene 35: 5170-5178, 2016.

11. Formolo CA, Williams R, Gordish-Dressman H, MacDonald TJ, Lee NH and Hathout Y: Secretome signature of invasive glioblastoma multiforme. J Proteome Res 10: 3149-3159, 2011.

12. Jongbloets BC, Ramakers GM and Pasterkamp RJ: Semaphorin7A and its receptors: Pleiotropic regulators of immune cell function, bone homeostasis, and neural development. Semin Cell Dev Biol 24: 129-138, 2013.

13. Fong KP, Barry C, Tran AN, Traxler EA, Wannemacher KM, Tang HY, Speicher KD, Blair IA, Speicher DW, Grosser T, et al: Deciphering the human platelet sheddome. Blood 117: e15-e26, 2011.

14. Holmes S, Downs AM, Fosberry A, Hayes PD, Michalovich D, Murdoch P, Moores K, Fox J, Deen K, Pettman G, et al: Sema7A is a potent monocyte stimulator. Scand J Immunol 56: 270-275, 2002.

15. Garcia-Areas R,Libreros S, Amat S, Keating P,Carrio R,Robinson P, Blieden $C$ and Iragavarapu-Charyulu V: Semaphorin7A promotes tumor growth and exerts a pro-angiogenic effect in macrophages of mammary tumor-bearing mice. Front Physiol 5: 17, 2014.

16. Liu H, Juo ZS, Shim AH, Focia PJ, Chen X, Garcia KC and He X: Structural basis of semaphorin-plexin recognition and viral mimicry from Sema7A and A39R complexes with PlexinC1. Cell 142: 749-761, 2010.

17. Kang HR, Lee CG, Homer RJ and Elias JA: Semaphorin 7A plays a critical role in TGF-betal-induced pulmonary fibrosis. J Exp Med 204: 1083-1093, 2007.

18. Suzuki K, Okuno T, Yamamoto M, Pasterkamp RJ, Takegahara N, Takamatsu H, Kitao T, Takagi J, Rennert PD, Kolodkin AL, et al: Semaphorin 7A initiates T-cell-mediated inflammatory responses through alphalbeta1 integrin. Nature 446: 680-684, 2007.

19. Markel P, Shu P, Ebeling C, Carlson GA, Nagle DL, Smutko JS and Moore KJ: Theoretical and empirical issues for markerassisted breeding of congenic mouse strains. Nat Genet 17: 280-284, 1997.

20. Wakeland E, Morel L, Achey K, Yui M and Longmate J: Speed congenics: A classic technique in the fast lane (relatively speaking). Immunol Today 18: 472-477, 1997.

21. Fellmann C, Hoffmann T, Sridhar V, Hopfgartner B, Muhar M, Roth M, Lai DY, Barbosa IA, Kwon JS, Guan Y, et al: An optimized microRNA backbone for effective single-copy RNAi. Cell Rep 5: 1704-1713, 2013.

22. Wojcikiewicz EP, Zhang X, Chen A and Moy VT: Contributions of molecular binding events and cellular compliance to the modulation of leukocyte adhesion. J Cell Sci 116: 2531-2539, 2003.

23. Hoh JH and Schoenenberger CA: Surface morphology and mechanical properties of MDCK monolayers by atomic force microscopy. J Cell Sci 107: 1105-1114, 1994.

24. Aslakson CJ and Miller FR: Selective events in the metastatic process defined by analysis of the sequential dissemination of subpopulations of a mouse mammary tumor. Cancer Res 52: 1399-1405, 1992.

25. Tao K, Fang M, Alroy J and Sahagian GG: Imagable 4T1 model for the study of late stage breast cancer. BMC Cancer 8: 228, 2008.

26. Ridley AJ: RhoA, RhoB and RhoC have different roles in cancer cell migration. J Microsc 251: 242-249, 2013.

27. Brown GT and Murray GI: Current mechanistic insights into the roles of matrix metalloproteinases in tumour invasion and metastasis. J Pathol 237: 273-281, 2015.

28. Xiang X, Zhuang X, Ju S, Zhang S, Jiang H, Mu J, Zhang L, Miller D, Grizzle W and Zhang HG: miR-155 promotes macroscopic tumor formation yet inhibits tumor dissemination from mammary fat pads to the lung by preventing EMT. Oncogene 30: 3440-3453, 2011.

29. Pickup M, Novitskiy S and Moses HL: The roles of TGF $\beta$ in the tumour microenvironment. Nat Rev Cancer 13: 788-799, 2013.

30. Padua D and Massagué J: Roles of TGFbeta in metastasis. Cell Res 19: 89-102, 2009.

31. Sánchez-Tilló E, Siles L, de Barrios O, Cuatrecasas M, Vaquero EC, Castells A and Postigo A: Expanding roles of ZEB factors in tumorigenesis and tumor progression. Am J Cancer Res 1: 897-912, 2011. 
32. Lamouille S, Xu J and Derynck R: Molecular mechanisms of epithelial-mesenchymal transition. Nat Rev Mol Cell Biol 15: $178-196,2014$.

33. Serrano-Gomez SJ, Maziveyi M and Alahari SK: Regulation of epithelial-mesenchymal transition through epigenetic and posttranslational modifications. Mol Cancer 15: 18, 2016.

34. Lekka M: Discrimination between normal and cancerous cells using AFM. Bionanoscience 6: 65-80, 2016.

35. Lekka M, Laidler P, Gil D, Lekki J, Stachura Z and Hrynkiewicz AZ: Elasticity of normal and cancerous human bladder cells studied by scanning force microscopy. Eur Biophys J 28: 312-316, 1999.

36. Cross SE, Jin YS, Rao J and Gimzewski JK: Nanomechanical analysis of cells from cancer patients. Nat Nanotechnol 2: 780-783, 2007.

37. De Minicis S, Rychlicki C, Agostinelli L, Saccomanno S, Trozzi L, Candelaresi C, Bataller R, Millán C, Brenner DA, Vivarelli M, et al: Semaphorin 7A contributes to TGF- $\beta$-mediated liver fibrogenesis. Am J Pathol 183: 820-830, 2013.

38. Saito T, Kasamatsu A, Ogawara K, Miyamoto I, Saito K, Iyoda M, Suzuki T, Endo-Sakamoto Y, Shiiba M, Tanzawa H, et al: Semaphorin7A promotion of tumoral growth and metastasis in human oral cancer by regulation of G1 cell cycle and matrix metalloproteases: Possible contribution to tumoral angiogenesis. PLoS One 10: e0137923, 2015.
39. Wetzig A, Alaiya A, Al-Alwan M, Pradez CB, Pulicat MS, Al-Mazrou A, Shinwari Z, Sleiman GM, Ghebeh H, Al-Humaidan H, et al: Differential marker expression by cultures rich in mesenchymal stem cells. BMC Cell Biol 14: 54, 2013.

40. Bongiorno T, Kazlow J, Mezencev R, Griffiths S, OlivaresNavarrete R, McDonald JF, Schwartz Z, Boyan BD, McDevitt TC and Sulchek T: Mechanical stiffness as an improved single-cell indicator of osteoblastic human mesenchymal stem cell differentiation. J Biomech 47: 2197-2204, 2014.

41. Guck J, Schinkinger S, Lincoln B, Wottawah F, Ebert S, Romeyke M, Lenz D, Erickson HM, Ananthakrishnan R, Mitchell D, et al: Optical deformability as an inherent cell marker for testing malignant transformation and metastatic competence. Biophys J 88: 3689-3698, 2005.

42. van Rijn A, Paulis L, te Riet J, Vasaturo A, Reinieren-Beeren I, van der Schaaf A, Kuipers AJ, Schulte LP, Jongbloets BC, Pasterkamp RJ, et al: Semaphorin 7A promotes chemokinedriven dendritic cell migration. J Immunol 196: 459-468, 2016. 